\title{
Immunohistochemical analysis of Reg IV in urogenital organs: Frequent expression of Reg IV in prostate cancer and potential utility as serum tumor marker
}

\author{
TETSUTARO HAYASHI ${ }^{1,2}$, AKIO MATSUBARA ${ }^{2}$, SHINYA OHARA $^{1,2}$, KOJI MITA $^{2}$, \\ YASUHISA HASEGAWA ${ }^{2}$, TSUGURU USUI ${ }^{2}$, KOJI ARIHIRO ${ }^{3}$, SHINICHI NORIMURA ${ }^{1}$, \\ KAZUHIRO SENTANI $^{1}$, NAOHIDE OUE ${ }^{1}$ and WATARU YASUI ${ }^{1}$
}

Departments of ${ }^{1}$ Molecular Pathology, ${ }^{2}$ Urology, Hiroshima University Graduate School of Biomedical Sciences;
${ }^{3}$ Department of Anatomical Pathology, Hiroshima University Hospital, Hiroshima, Japan

Received June 25, 2008; Accepted August 26, 2008

DOI: 10.3892/or_00000194

\begin{abstract}
Regenerating islet-derived family, member 4 (Reg IV) is a candidate marker for cancer and inflammatory bowel disease and is associated with neuroendocrine and intestinal differentiation. We have reported that $14 \%$ of prostate cancer (PCa) cases are positive for Reg IV by immunohistochemistry. In the present study, we performed immunohistochemical analysis of Reg IV in other major urological cancers, including 101 renal cell carcinoma (RCC), and 95 urothelial carcinoma (UC) of urinary bladder by immunohistochemistry. We also investigated neuroendocrine differentiation by chromogranin A and synaptophysin staining along with intestinal differentiation by MUC2 staining. Immunohistochemical analysis of Reg IV revealed no expression of Reg IV in RCC, and only one case (1\%) of UC expressed Reg IV. Neither neuroendocrine nor intestinal differentiation was found in RCC. Among 95 UC cases, neuroendocrine differentiation was detected in 13 cases $(14 \%)$, and intestinal differentiation was observed in 33 cases $(35 \%)$. In one Reg IV-positive UC case, MUC2 staining was observed. Since Reg IV expression was frequently found in $\mathrm{PCa}$, we also measured Reg IV levels in sera from patients with PCa by enzyme-linked immunosorbent assay. The serum Reg IV concentration in PCa patients $(\mathrm{n}=38$, mean $\pm \mathrm{SE}, 1.69 \pm 0.16 \mathrm{ng} / \mathrm{ml})$ was significantly higher than that in control individuals $(n=40,1.28 \pm 0.11$ $\mathrm{ng} / \mathrm{ml}, \mathrm{P}=0.0199$, Mann-Whitney U test). The sensitivity and specificity for detection of PCa were 34\% (13/38) and 90\% (36/40), respectively. These results suggest that among
\end{abstract}

Correspondence to: Dr Wataru Yasui, Department of Molecular Pathology, Hiroshima University Graduate School of Biomedical Sciences, 1-2-3 Kasumi, Minami-ku, Hiroshima 734-8551, Japan E-mail: wyasui@hiroshima-u.ac.jp

Key words: regenerating islet-derived family member 4, serum tumor marker, prostate cancer major urologic cancers, Reg IV is expressed frequently in $\mathrm{PCa}$, and that serum Reg IV represents a novel biomarker for PCa.

\section{Introduction}

Prostate cancer (PCa) is one of the most common types of cancer and is the second leading cause of cancer death among men in the United States (1). Currently, the standard diagnostic marker for $\mathrm{PCa}$ is prostate-specific antigen (PSA) and the rapid incorporation of aggressive PSA testing has resulted in dramatically earlier identification of PCa (2). However, the significantly high false-positive rate of PSA combined with its widespread clinical application, has lead to a tremendous increase in the number of unnecessary prostate biopsies (3). Therefore, there has been an increasing emphasis in the need to determine multiple protein biomarkers for use in the diagnosis and prognosis of PCa.

Renal cell carcinoma (RCC) accounts for $\sim 2 \%$ of all human cancers worldwide, and in 2000189,000 cases and 91,000 deaths were reported (4). RCC is characterized by an absence of early warning signs leading to a rather high percentage of advanced, metastatic tumors upon diagnosis. For example, the classic triad of pain, hematuria, and a palpable flank mass is encountered in only $10 \%$ of patients and is usually associated with the presence of advanced disease (5). Unlike other solid malignancies in which established serum or urinary biomarkers are available for early detection, relatively few diagnostic tools are currently available for the early detection of renal tumors. Therefore, an ideal tumor marker with high sensitivity and specificity offers the ideal opportunity for early detection of RCC.

Bladder cancer, the majority of which is urothelial carcinoma (UC) is the sixth most common cancer in the world. A distinctive feature of UC is that multiple metachronous or synchronous cancers frequently develop, arising from either a polyclonal origin or metastasis from a single clone. Thus, bladder cancer patients need a long-term followup with repeated urine cytology and cystoscopy for monitoring. Conventional urine cytology has been the standard noninvasive method for cancer detection and disease monitoring. 
However, the sensitivity of this method is known to be low, especially for low-grade UC. Therefore, a more sensitive, non-invasive method for cancer detection is required.

We previously performed serial analysis of gene expression (SAGE) of four primary gastric cancers (6) and identified several gastric cancer-specific genes (7). Of these genes, regenerating islet-derived family, member 4 (REG4, which encodes Reg IV) is a candidate gene for cancer-specific expression, at least in patients with gastric cancer. REG4 is a member of the REG gene family, which includes three other genes, and was originally identified by high-throughput sequence analysis of a large inflammatory bowel disease cDNA library (8). Quantitative reverse transcription polymerase chain reaction (RT-PCR) analysis revealed that $\sim 50 \%$ of gastric cancers overexpress the REG4 gene (6) and Reg IV expression is associated with the intestinal and neuroendocrine differentiation (9). In addition to gastric cancer, overexpression of Reg IV in colorectal cancer (10), pancreatic cancer (11) and PCa (12) has been reported. In our previous immunohistochemical analysis (13), $14 \%$ of the PCa cases were positive for Reg IV and Reg IV positivity was associated with intestinal and neuroendocrine differentiation. Furthermore, Reg IV expression is an independent prognostic indicator of relapse after radical prostatectomy. However, histologically, gastric, colorectal, pancreatic and prostate cancers are adenocarcinomas, and expression of Reg IV except for adenocarcinomas, such as RCC or UC, has not yet been investigated.

Reg IV is a secreted protein which we have previously shown represents a novel biomarker for gastric cancer (14). The diagnostic sensitivity of serum Reg IV was superior to that of serum carcinoembryonic antigen or carbohydrate antigen 19-9. Serum Reg IV serves as a tumor marker for colorectal and pancreatic cancer $(10,11)$. These data support the idea that Reg IV protein has potential as a novel serum tumor marker for a wide variety of malignancies; however, serum concentration of Reg IV in major urologic cancers including PCa, RCC or UC has not previously been measured.

In the present study, we examined the expression and distribution of Reg IV in human RCC and UC by immunohistochemistry. We have reported two Reg IV staining patterns (mucin-like and perinuclear staining) (9). Mucin-like Reg IV staining, observed in goblet cells and goblet cell-like vesicles of tumor cells, is associated with MUC2 (a goblet cell marker) positivity. Perinuclear Reg IV staining is detected in cells with neuroendocrine differentiation. Therefore, we also performed immunohistochemical analysis of MUC2, chromogranin A (a neuroendocrine cell marker) and synaptophysin (a neuroendocrine cell marker). Since Reg IV expression was frequently found in PCa, we also measured Reg IV levels in sera from patients with PCa by enzyme-linked immunosorbent assay (ELISA) to investigate the potential diagnostic utility of Reg IV measurement.

\section{Materials and methods}

Tissue samples. In total, 204 primary tumor samples and 78 serum samples were collected. Patients were treated at the Hiroshima University Hospital or an affiliated hospital.

For immunohistochemical analysis, we used archival formalin-fixed, paraffin-embedded tissues from 196 patients who had undergone surgical excision for either RCC $(n=101)$ or UC ( $\mathrm{n}=95)$. All 101 patients with RCC were treated by radical nephrectomy, and all 95 patients with UC were treated by cystectomy. Tumor staging was performed according to the TNM classification system (15).

For Western blot analysis, 8 RCC samples and the corresponding non-neoplastic kidney samples were used. The samples were obtained during surgery at Hiroshima University Hospital or an affiliated hospital. We confirmed microscopically that the tumor specimens were predominantly RCC tissue ( $>80 \%)$. Samples were frozen immediately in liquid nitrogen and stored at $-80^{\circ} \mathrm{C}$ until use.

All serum samples were collected before prostate biopsy and stored at $-80^{\circ} \mathrm{C}$. Seventy-eight consecutive men who visited the outpatient clinic of the Department of Urology, Hiroshima University Hospital due to elevated PSA levels $(>4.0 \mathrm{ng} / \mathrm{ml})$ served as the study population. The PCa population consisted of 38 men with evidence of cancer from a prostate biopsy (age range 50-93 years, mean 73 years), and the remaining 40 men without evidence of cancer from a prostate biopsy served as a control population (age range 4790 years, mean 66 years). In accordance with the Ethics Guidelines for Human Genome/Gene Research enacted by the Japanese Government, tissue specimens were collected and used on the basis of approval from the Ethics Review Committee of the Hiroshima University School of Medicine and from the ethics review committees of collaborating organizations.

Immunohistochemistry. Formalin-fixed, paraffin-embedded samples were sectioned, deparaffinized and stained with $H \& E$ to ensure that the sectioned block contained tumor cells. Adjacent sections were then immunohistochemically stained. Antigen retrieval was performed by microwave heating in citrate buffer ( $\mathrm{pH}$ 6.0) for $30 \mathrm{~min}$ for Reg IV, MUC2, chromogranin A and synaptophysin. Peroxidase activity was blocked with $3 \% \mathrm{H}_{2} \mathrm{O}_{2}$-methanol for 10 min and sections were then incubated with normal goat serum (Dako Cytomation, Carpinteria, CA, USA) for 20 min to block nonspecific antibody binding. Sections were incubated with a primary antibody against Reg IV (rabbit polyclonal antibody, diluted 1:50; anti-Reg IV antibody was raised and characterized in our laboratory) (9), MUC2 (1:50; Novocastra, Newcastle, UK), chromogranin A (1:50; Novocastra), or synaptophysin (1:50; Dako Cytomation) for $1 \mathrm{~h}$ at room temperature, followed by incubation with peroxidase-labeled anti-rabbit or anti-mouse IgG for $1 \mathrm{~h}$. Staining was completed with a 10 -min incubation in a substrate-chromogen solution. The sections were counterstained with $0.1 \%$ hematoxylin. The specificity of the Reg IV antibody has been characterized in detail (9). Staining of each antibody was considered positive if any tumor cells were stained. Intestinal differentiation was defined as positive staining for MUC2. Neuroendocrine differentiation was defined as positive staining for chromogranin A and/or synaptophysin.

Western blot analysis. Preparation of whole cell lysates from the RCC samples and Western blotting were performed as previously described (16). Protein concentrations were determined by Bradford protein assay (Bio-Rad, Richmond, 

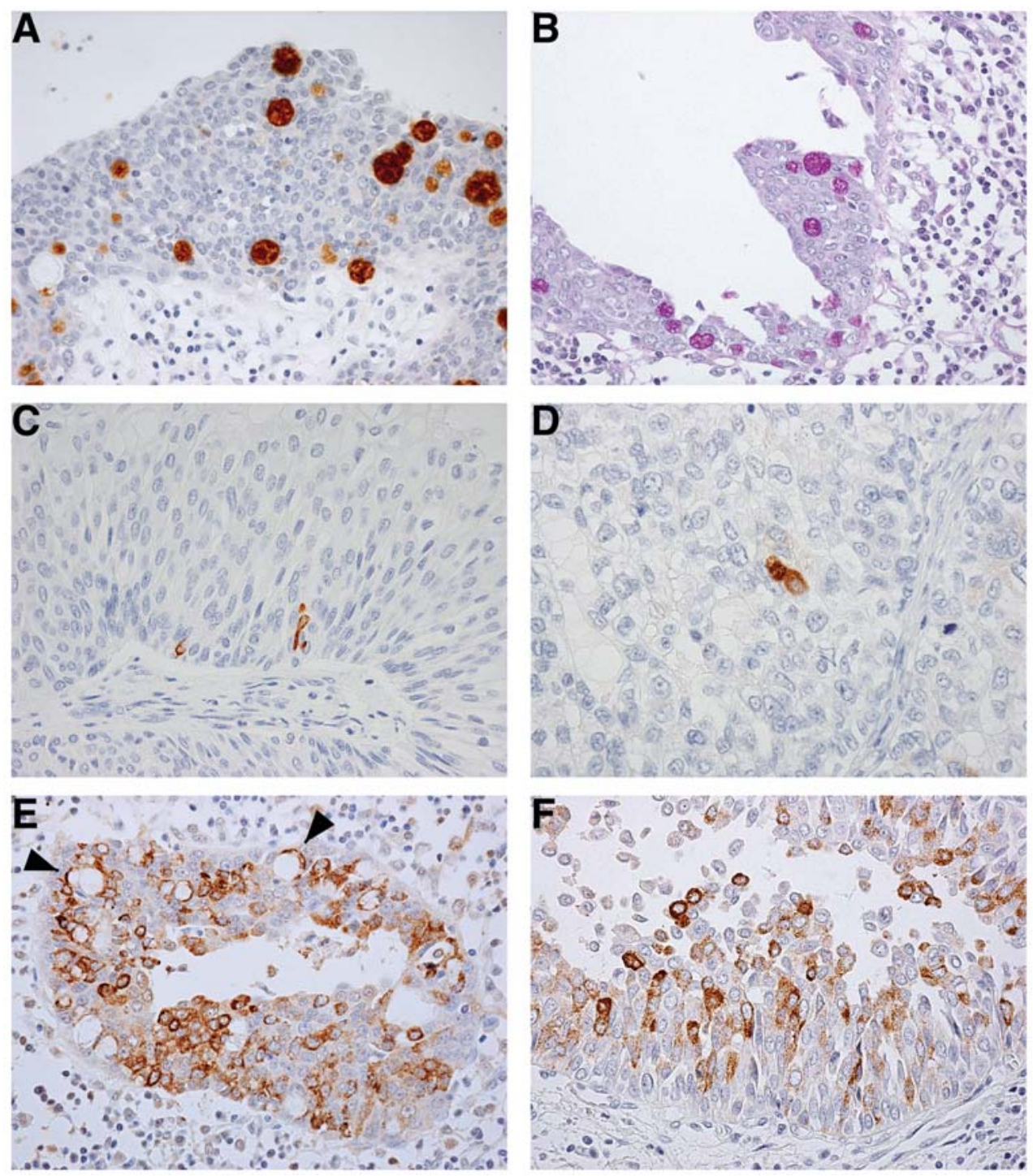

Figure 1. Immunohistochemical analysis of Reg IV expression in UC of the urinary bladder. (A) Immunostaining of Reg IV in UC. In case 37, mucin-like staining of Reg IV is present in goblet cell-like vesicles of tumor cells. (B) In case 37, PAS staining was observed in gland-like lumina. (C) Immunostaining of chromogranin A in UC. (D) Immunostaining of synaptophysin in UC. (E) Immunostaining of MUC2 in UC. In case 37, mucin-like and perinuclear staining are observed in tumor cells. Arrowhead indicates mucin-like staining of MUC2. (F) Immunostaining of MUC2 in UC. Perinuclear staining of MUC2 is present in tumor cells.

CA, USA) with BSA used as the standard. Protein (20 $\mu \mathrm{g}$ per lane) was electrophoresed on SDS-PAGE gels and transferred to nitrocellulose filters. Filters were incubated for $1 \mathrm{~h}$ at room temperature with anti-Reg IV antibody (rabbit polyclonal antibody raised in our laboratory). Peroxidase conjugated anti-rabbit IgG was used in the secondary reaction. The immunocomplexes were visualized with an ECL Western blot detection system (Amersham Biosciences, Piscataway, NJ, USA). The quality and amounts of proteins on the gel were confirmed by detection with anti- $\beta$-actin antibody (Sigma-Aldrich, St. Louis, MO, USA).

ELISA. For measurement of the serum Reg IV concentration, a sandwich ELISA method was developed as previously described (14). First, polystyrene microtiter plates were coated with mouse monoclonal anti-Reg IV antibody (R\&D Systems, Abingdon, UK) by overnight incubation of $50 \mu \mathrm{l} /$
$125 \mathrm{ng}$ per well of antibody diluted in Tris buffer ( $\mathrm{pH} 7.4$ ). The plates were then washed 3 times with wash buffer. After the plates were blocked with $1 \%$ milk in phosphate-buffered saline, $50 \mu \mathrm{l}$ of recombinant Reg IV standard or sample was added to each well and incubated overnight at $4^{\circ} \mathrm{C}$. After 3 washes, $50 \mu \mathrm{l}$ of biotinylated goat polyclonal anti-Reg IV antibody (R\&D Systems) in assay buffer [1\% BSA, Tris buffer (pH 7.4) and $0.05 \%$ normal goat serum] was added to each well (75 $\mathrm{ng}$ antibody per well). The mixture was then incubated for $1 \mathrm{~h}$ with shaking at $37^{\circ} \mathrm{C}$ and washed 3 times with wash buffer. The plates were incubated with $50 \mu 1$ per well alkaline phosphatase-conjugated streptavidin (Dako) diluted 1:2000 in diluent containing 1\% BSA and Tris buffer ( $\mathrm{pH} \mathrm{7.4)} \mathrm{for} 1 \mathrm{~h}$ at $37^{\circ} \mathrm{C}$ and washed 3 times. Color development was performed with the addition of pNPP chromogenic substrate (Sigma-Aldrich) followed by incubation at $37^{\circ} \mathrm{C}$ for $1 \mathrm{~h}$. Absorbance at $405 \mathrm{~nm}$ was measured with an ELISA 

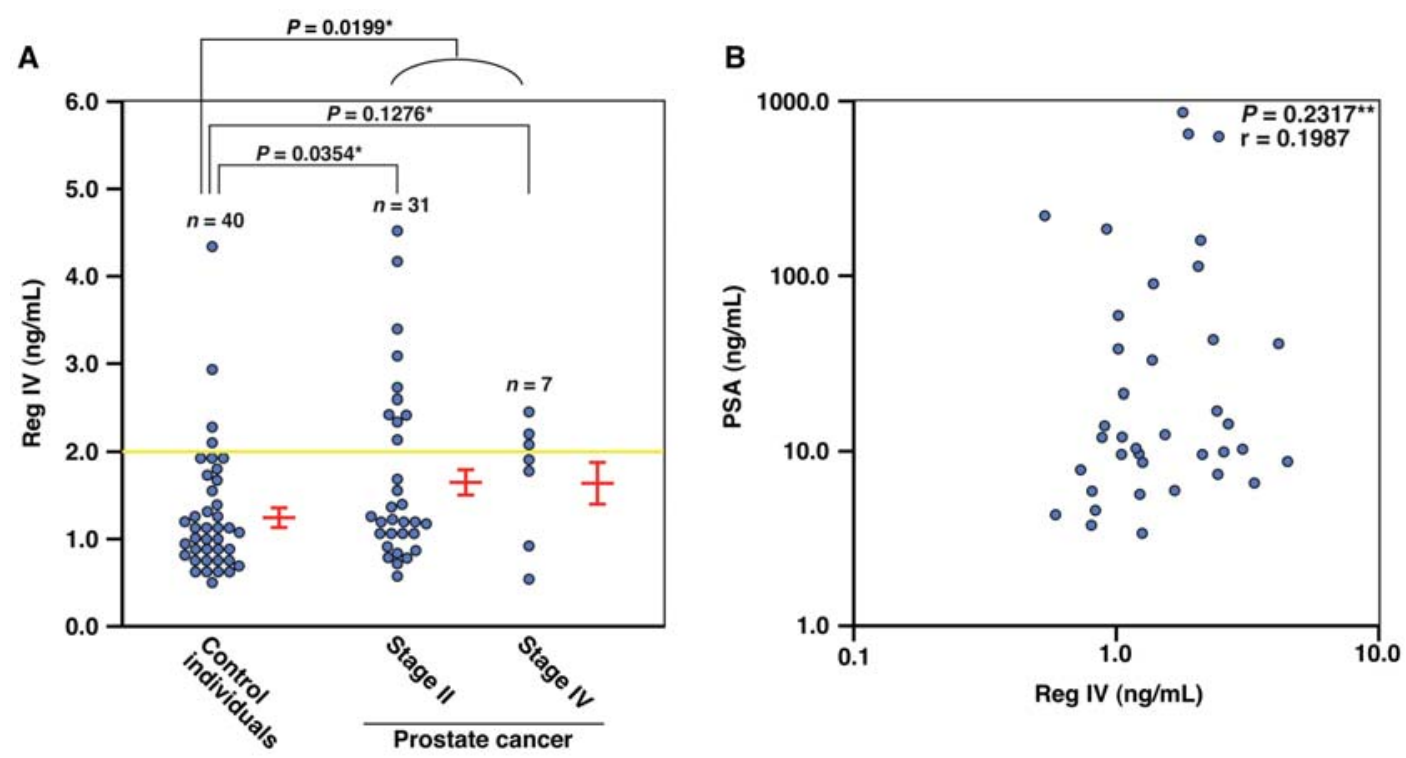

Figure 2. ELISA of serum samples from 40 control individuals and 38 patients with PCa. (A) Detection of Reg IV protein in serum samples by ELISA. Yellow bars indicate the cut-off levels defined on the basis of the previous study [2.00 ng/ml (14)]. Red bars indicate the mean \pm SE. Differences in the serum concentration of Reg IV between two groups are tested by Mann-Whitney U test (*). (B) Relation between serum concentrations of Reg IV and PSA. Correlation is examined using Spearman's rank correlation $(* *)$.

plate reader. As a reference standard, known concentrations of human recombinant Reg IV (9) from 0 to $30 \mathrm{ng} / \mathrm{ml}$ were tested in triplicate.

Statistical methods. Association between clinicopathologic variables and Reg IV expression was analyzed by Fisher's exact test. Differences in the serum Reg IV concentration between the two groups were tested by the non-parametric Mann-Whitney U test. Correlations between the serum Reg IV concentration and the serum concentration of PSA were assessed by Spearman's rank correlation test. $\mathrm{P}<0.05$ was considered statistically significant.

\section{Results}

Expression of Reg IV and neuroendocrine and intestinal differentiation in $R C C$. We performed immunohistochemical analysis of Reg IV in 101 RCC samples; however, no staining of Reg IV was found. In adjacent non-neoplastic kidney tissues, no Reg IV staining was found. Western blot analysis of Reg IV was also performed in 8 RCC samples (data not shown), in which no Reg IV expression was confirmed in the RCC samples nor in adjacent non-neoplastic kidney samples. We also performed immunohistochemical analysis of chromogranin A, synaptophysin and MUC2; however, no staining was detected.

Expression of Reg IV and neuroendocrine and intestinal differentiation in $U C$. We performed immunohistochemical analysis of Reg IV in 95 UC samples. In adjacent nonneoplastic urinary bladder tissues, no Reg IV staining was found. In UC tissues, Reg IV staining was observed in 1 (1\%) of 95 UC cases. In this Reg IV-positive UC case (case 37), Reg IV-positive tumor cells were observed in $10 \%$ of tumor cells, and mucin-like staining (Fig. 1A) was observed. In case 37, Reg IV staining was observed in gland like lumina, and periodic acid-Schiff (PAS) staining was also observed in gland-like lumina (Fig. 1B), indicating that this Reg IV-positive case represents glandular differentiation. Analysis of the relationship between Reg IV staining and clinicopathologic characteristics showed that Reg IV did not correlate with gender, age, $\mathrm{pT}$ category, $\mathrm{pN}$ category or tumor stage (data not shown).

Immunostaining of chromogranin A and synaptophysin was also performed. Representative results of chromogranin A and synaptophysin immunostaining in UC are shown in Fig. 1C and D, respectively. Of the 95 UC cases, chromo-granin A-positive cases were observed in 3 cases (3\%) and synaptophysin-positive cases were found in 11 cases $(12 \%)$. In total, UC cases showing neuroendocrine differentiation were found in 13 cases (14\%). However, case 37 (Reg IV-positive UC case) did not show neuroendocrine differentiation. Analysis of the relationship between neuroendocrine differentiation and clinicopathologic characteristics showed no correlation between neuroendocrine differentiation and gender, age, pT category, $\mathrm{pN}$ category or tumor stage (data not shown).

In UC tissues, MUC2 staining was observed in goblet cell-like vesicles (Fig. 1E) and perinuclear regions (Fig. 1F) of tumor cells. Of the $95 \mathrm{UC}$ cases, MUC2-positive cases were observed in 33 cases (35\%). Among the $33 \mathrm{UC}$ cases showing positive results for MUC2, one case, case 37 (Reg IVpositive UC case), showed MUC2 staining in goblet cell-like vesicles and perinuclear regions. The remaining 32 cases showed only perinuclear MUC2 staining. Analysis of the relationship between MUC2 positivity and clinicopathologic characteristics showed no correlation between MUC2positivity and gender, age, $\mathrm{pT}$ category, $\mathrm{pN}$ category or tumor stage (data not shown).

Serum Reg IV concentration in patients with PCa. As Reg IV expression was frequently found in $\mathrm{PCa}$, we also measured 
Reg IV levels in sera from patients with PCa by ELISA to investigate the potential diagnostic utility of Reg IV measurement. Serum Reg IV levels in 40 control individuals and 38 patients with PCa prior to biopsy are shown in Fig. 2A. The serum Reg IV concentration in PCa patients $(n=38$, $1.69 \pm 0.16 \mathrm{ng} / \mathrm{ml}$, mean $\pm \mathrm{SE}$ ) was significantly higher than that in control individuals $(\mathrm{n}=40,1.28 \pm 0.11 \mathrm{ng} / \mathrm{ml}, \mathrm{P}=0.0199$, Mann-Whitney U test), even at stage II $(n=31,1.69 \pm 0.18 \mathrm{ng} / \mathrm{ml}$, $\mathrm{P}=0.0354$, Mann-Whitney U test) (Fig. 2A). The mean of serum Reg IV concentration in PCa patients at stage IV $(n=7$, $1.68 \pm 0.26 \mathrm{ng} / \mathrm{ml}$ ) was higher than that in control individuals $(\mathrm{n}=40,1.28 \pm 0.11 \mathrm{ng} / \mathrm{ml})$; however, statistical difference was not found ( $\mathrm{P}=0.1276$, Mann-Whitney $\mathrm{U}$ test). The Reg IV concentration in serum samples from patients with $\mathrm{PCa}$ showing a Gleason score of $\leq 7(\mathrm{n}=22,1.75 \pm 0.21 \mathrm{ng} / \mathrm{ml})$ was not significantly different from those showing a Gleason score of $\geq 8(n=16,1.60 \pm 0.24 \mathrm{ng} / \mathrm{ml})(P=0.7448$, MannWhitney $\mathrm{U}$ test). In our previous study, the cut-off level for Reg IV was set at $2.00 \mathrm{ng} / \mathrm{ml}$ (14). In the group showing serum PSA levels of $>4 \mathrm{ng} / \mathrm{ml}$, the sensitivity and specificity for detection of PCa were 34 (13/38) and 90\% (36/40), respectively. Spearman's rank correlation test revealed that significant correlation was not found between serum Reg IV and PSA levels ( $\mathrm{r}=0.1987, \mathrm{P}=0.2317$ ) (Fig. 2B).

\section{Discussion}

Previously, we showed that $14 \%$ of the PCa cases were positive for Reg IV, and Reg IV positivity was associated with intestinal and neuroendocrine differentiation (13). These PCa cases were all adenocarcinomas. In the present study, immunohistochemical analysis of Reg IV was performed in other major urologic cancers including RCC and UC, both of which are not adenocarcinomas. Immunohistochemical analysis of Reg IV revealed that Reg IV expression was not found in RCC, and only $1 \%$ of UC expressed Reg IV. These results and our previous immunohistochemical analysis of Reg IV in PCa indicate that Reg IV is expressed frequently in PCa among major urologic cancers. Furthermore, we showed that the serum Reg IV concentration in PCa patients was significantly higher than that in control individuals. The sensitivity of serum Reg IV concentration was $34 \%$, and the specificity was $90 \%$. It has been reported that the specificity of the PSA test is only $20 \%$ at a sensitivity of $80 \%$ (17), indicating that serum Reg IV concentration serve as a diagnostic tumor marker with high specificity. Since serum Reg IV concentration was measured in the group showing serum PSA levels of $>4 \mathrm{ng} / \mathrm{ml}$, the sensitivity and specificity calculated in the present study may differ from those in healthy individuals, and serum concentration of Reg IV should also be measured in the group showing serum PSA levels of $<4 \mathrm{ng} / \mathrm{ml}$.

In urologic cancers including $\mathrm{PCa}, \mathrm{RCC}$ and $\mathrm{UC}$, urine represents a particularly useful fluid in which to examine tumor markers because of its enhanced potential to contain higher concentrations of directly released tumor-derived products and the fact that collection is non-invasive. In the present study, Reg IV was detected in serum samples from patients with PCa. Reg IV expression was not found in RCC, and only $1 \%$ of UC expressed Reg IV. In adjacent non- neoplastic kidney and urinary bladder tissues, no Reg IV staining was found. These results suggest that Reg IV concentration in urine may represent a novel marker for $\mathrm{PCa}$ at high specificity.

In RCC, no Reg IV expression was detected. Although neuron-specific enolase (a neuroendocrine cell marker) is frequently expressed in RCC, chromogranin A expression is rare (18). It has been reported that staining of MUC2 is not found in 16 RCC cases (19). In the present study, we also observed that RCC showed neither neuroendocrine differentiation based on chromogranin A and synaptophysin staining nor MUC2 staining. As Reg IV expression is associated with neuroendocrine and intestinal differentiation, these results are accordance with immunonegativity of Reg IV in RCC.

In UC, only 1 (1\%) UC case showed Reg IV staining. In the Reg IV-positive UC case, Reg IV staining was observed in gland-like lumina. Although this case was UC, PAS staining was also observed in gland-like lumina, indicating that this Reg IV-positive case possessed glandular differentiation. These results indicate that conventional UC is negative for Reg IV; however, UC with glandular differentiation may express Reg IV. A previous immunohistochemical study demonstrated that expression of chromogranin A is seen in $63.5 \%$ of UC cases (20). Yet, in the present study, only $14 \%$ of UC cases showed neuroendocrine differentiation and did not exhibit Reg IV expression. However, in gastric cancer, chromogranin A-positive tumor cells are not always positive for Reg IV (9). Furthermore, the one UC case with glandular differentiation was positive for MUC2 in goblet cell-like vesicles. Yet, Reg IV staining was not found in UC cases showing perinuclear MUC2 staining, nor was glandular differentiation observed in these cases. Based on this evidence, Reg IV expression is not likely involved in neuroendocrine differentiation of UC but is likely associated with glandular differentiation.

In conclusion, we showed that serum Reg IV concentration serves as a diagnostic tumor marker with high specificity for PCa. As the significantly high false-positive rate of PSA has lead to a tremendous increase in the number of unnecessary biopsies of the prostate (3), measurement of serum concentration of Reg IV may decrease the number of unnecessary prostate biopsies. Reg IV is correlated with relapse-free survival of patients with PCa (13), and hormone-refractory $\mathrm{PCa}$ has been reported to express high levels of REG4 mRNA (12). Therefore, serum concentration of Reg IV may predict relapse-free survival and resistance to androgendeprivation therapy.

\section{Acknowledgements}

We thank Mrs Emiko Hisamoto for excellent technical assistance and advice. This work was supported, in part, by Grants-in-Aid for Cancer Research from the Ministry of Education, Culture, Science, Sports and Technology of Japan and from the Ministry of Health, Labor and Welfare of Japan. This work was carried out with the kind cooperation of the Research Center for Molecular Medicine, Faculty of Medicine, Hiroshima University. We thank the Analysis Center of Life Science, Hiroshima University, for the use of their facilities. 


\section{References}

1. Jemal A, Siegel R, Ward E, Murray T, Xu J and Thun MJ: Cancer statistics. CA Cancer J Clin 57: 43-66, 2007.

2. Carter HB: Prostate cancers in men with low PSA levels - must we find them? N Engl J Med 350: 2292-2294, 2004.

3. Gambert SR: Prostate cancer. When to offer screening in the primary care setting. Geriatrics 56: 22-26, 2001.

4. Parkin DM, Bray F, Ferlay J and Pisani P: Estimating the world cancer burden: Globocan 2000. Int J Cancer 94: 153-156, 2001.

5. Pantuck AJ, Zisman A, Rauch MK and Belldegrun A: Incidental renal tumors. Urology 56: 190-196, 2000.

6. Oue N, Hamai Y, Mitani Y, et al: Gene expression profile of gastric carcinoma: identification of genes and tags potentially involved in invasion, metastasis, and carcinogenesis by serial analysis of gene expression. Cancer Res 64: 2397-2405, 2004.

7. Aung PP, Oue N, Mitani Y, et al: Systematic search for gastric cancer-specific genes based on SAGE data: melanoma inhibitory activity and matrix metalloproteinase-10 are novel prognostic factors in patients with gastric cancer. Oncogene 25: 2546-2557, 2006.

8. Hartupee JC, Zhang H, Bonaldo MF, Soares MB and Dieckgraefe BK: Isolation and characterization of a cDNA encoding a novel member of the human regenerating protein family: Reg IV. Biochim Biophys Acta 1518: 287-293, 2001.

9. Oue N, Mitani Y, Aung PP, et al: Expression and localization of Reg IV in human neoplastic and non-neoplastic tissues: Reg IV expression is associated with intestinal and neuroendocrine differentiation in gastric adenocarcinoma. J Pathol 207: 185-198, 2005.

10. Oue N, Kuniyasu H, Noguchi T, et al: Serum concentration of Reg IV in patients with colorectal cancer: overexpression and high serum levels of Reg IV are associated with liver metastasis. Oncology 72: 371-380, 2007.

11. Takehara A, Eguchi H, Ohigashi $\mathrm{H}$, et al: Novel tumor marker REG4 detected in serum of patients with resectable pancreatic cancer and feasibility for antibody therapy targeting REG4. Cancer Sci 97: 1191-1197, 2006.
12. Gu Z, Rubin MA, Yang Y, et al: Reg IV: a promising marker of hormone refractory metastatic prostate cancer. Clin Cancer Res 11: 2237-2243, 2005

13. Ohara S, Oue N, Matsubara A, et al: Reg IV is an independent prognostic factor for relapse in patients with clinically localized prostate cancer. Cancer Sci (In press).

14. Mitani Y, Oue N, Matsumura S, et al: Reg IV is a serum biomarker for gastric cancer patients and predicts response to 5fluorouracil-based chemotherapy. Oncogene 26: 4383-4393, 2007.

15. Sobin LH and Wittekind CH (eds): TNM Classification of Malignant Tumors, 6th edition. Wiley-Liss, New York, pp184-202, 2002.

16. Yasui W, Ayhan A, Kitadai Y, et al: Increased expression of p34cdc2 and its kinase activity in human gastric and colonic carcinomas. Int J Cancer 53: 36-41, 1993.

17. Catalona WJ, Hudson MA, Scardino PT, et al: Selection of optimal prostate specific antigen cutoffs for early detection of prostate cancer: receiver operating characteristic curves. J Urol 152: 2037-2042, 1994

18. Rasmuson T, Grankvist K, Roos G and Ljungberg B: Neuroendocrine differentiation in renal cell carcinoma-evaluation of chromogranin A and neuron-specific enolase. Acta Oncol 38: 623-628, 1999.

19. Lau SK, Weiss LM and Chu PG: Differential expression of MUC1, MUC2, and MUC5AC in carcinomas of various sites: an immunohistochemical study. Am J Clin Pathol 122: 61-69, 2004.

20. Mai KT, Perkins DG, Parks W, Rippstein P and Farmer J: Unusual immunostaining pattern of chromogranin in normal urothelium and in transitional cell neoplasms. Acta Histochem 96: 303-308, 1994. 